\section{MCL1's resistance story}

\section{By Michael J. Haas, Senior Writer}

Separate studies by Harvard Medical School and Roche's Genentech Inc. unit have shown that increased levels of myeloid leukemia cell differentiation protein, an antiapoptotic protein in the B cell lymphoma 2 family, can enable acute lymphoblastic leukemia cells and a variety of solid tumors to resist antitubulin agents. ${ }^{1,2}$ The findings suggest that profiling levels of the protein in patients could help determine whether chemotherapy will be effective.

In addition, companies with $B$ cell lymphoma 2 (BCL-2; BCL2) inhibitors that have myeloid leukemia cell differentiation protein (MCL1) activity may consider pairing their molecules with antitubulin agents to try and elicit synergistic activity.

The BCL2 family of proteins includes multiple anti- and proapoptotic factors that regulate survival and death in normal cells. Cancer cells can overexpress more than one antiapoptotic protein of the BCL2 family. MCL1 is one such protein, but the specific cancers in which MCL1 is the primary driver of survival and the mechanism by which MCL1 expression is upregulated have been unclear.

The teams arrived at their conclusions about MCL1's role in cancer from different starting points. The Harvard team was elucidating the mechanism by which deficiencies in the tumor suppressor FBW7 (f-box and WD repeat domain containing 7; FBXW7) give rise to acute lymphoblastic leukemia (ALL), whereas the Genentech team was studying how antitubulin agents induce apoptosis in solid tumors and the mechanisms that tumors develop to resist those drugs.

"Each group was asking very different questions in different systems," said Genentech team leader Ingrid Wertz, a research scientist in the company's Department of Early Discovery Biochemistry. "Yet we arrived at the same conclusions, thus providing each team with independent validation of its findings."

"What we find most valuable about the papers is simply the additional evidence that loss of MCL1 function can be an effective method for killing cancer cells as a single agent or in combination therapies," said Anthony Letai, cofounder and chairman of the scientific advisory board for Eutropics Pharmaceuticals Inc. "The pursuit of drugs that inhibit MCL1 function has been an active area of investigation by several companies and laboratories. The results in the Nature papers offer further support for this strategy."

\section{ALL aboard}

Harvard researchers led by Wenyi Wei, assistant professor of pathology at Beth Israel Deaconess Medical Center, initially wanted to know how FBW7 contributes to ALL pathogenesis because FBW7 deletions or inactivating mutations occur in $20 \%-30 \%$ of ALL cells. ${ }^{3}$ Moreover, knocking out $F b w 7$ in mouse T cells can cause ALL. ${ }^{4}$

FBW7 is a substrate-binding component of the E3 ubiquitin ligase complex and targets oncogenic proteins for degradation by the proteasome, such as c-jun $\mathrm{N}$-terminal kinase (JNK), c-Myc and Notch 1 (NOTCH1).

In addition to ALL, FBW7 deficiencies have been found in multiple solid tumors. ${ }^{5}$ However, the precise role FBW7 plays in promoting cancer growth and survival is not well understood because several of its targets can promote either cellular proliferation or apoptosis under different conditions.

Wei's team found that ALL cells deficient in FBW7 had higher levels of MCL1 than wildtype ALL cells. Deficient cells treated with ABT-737, which inhibits several BCL2 family proteins but does not have an effect on MCL1, showed less apoptosis than wild-type cells.

Nexavar sorafenib, which inhibits MCL1 in addition to a handful of receptor tyrosine kinases, restored the deficient cells' sensitivity to ABT-737.

Onyx Pharmaceuticals Inc. and Bayer AG market Nexavar to treat liver and renal cancers.

ABT-737 is a research compound from Abbott Laboratories. The company discontinued development of the compound after preclinical studies showed it had poor oral bioavailability. ${ }^{6}$

In addition to Harvard, Wei's team included researchers from Brigham and Women's Hospital, Dana-Farber Cancer Institute, The Jackson Laboratory, Massachusetts General Hospital Cancer Center and Kyushu University.

Wei's group is investigating whether FBW7-deficient ALL cells are more sensitive to sorafenib monotherapy than wild-type ALL cells. "We are also continuing to investigate whether genetic loss of FBW7 in ALL cells is the primary cause of resistance to ABT-737" in human ALL cell lines, he said.

Wei said Beth Israel has filed a patent application covering his team's finding and the IP is available for licensing or partnering.

\section{Different track}

In contrast to the Harvard team, the Genentech team wanted to know how antitubulin agents kill solid tumor cells and how tumors develop resistance to those drugs. The team focused on antiapoptotic BCL2 family proteins because of their known roles in tumorigenesis and chemotherapy resistance. ${ }^{?}$ 
First, Wertz's team knocked out members of the BCL2 family one at a time from mouse embryonic fibroblasts and myeloid cell lines and then treated the cells with either paclitaxel or vincristine. Cells deficient in Mcll were more sensitive to the drugs than wild-type cells or cells deficient in other antiapoptotic BCL2 proteins such as Bcl- $\mathrm{x}_{1}$.

Next, the team investigated the mechanisms regulating MCL1 levels in wild-type human cancer cell lines and found that FBW7 targeted MCL1 for degradation. Moreover, ovarian and colon cancer cell lines lacking FBW7 had higher MCL1 levels and were consequently less sensitive to paclitaxel and vincristine than wild-type controls.

In mice with ovarian xenograft tumors, paclitaxel or vincristine was less effective at decreasing the growth of FBW7-deficient tumors than of wild-type tumors. Treatment of the FBW7-deficient models with MCL1 small hairpin RNA restored the tumors' sensitivity to the drugs.

Finally, the team identified an association between FBW7 deficiency and increased MCL1 levels in primary ovarian and non-small cell lung cancer (NSCLC) tumors-both of which are frequently treated with antitubulin agents.

Wertz's team included researchers from The Walter and Eliza Hall Institute of Medical Research, The University of Melbourne and Abbott.

The findings identify "one more piece of the puzzle of chemotherapy response and one important mechanism of drug resistance" and suggest that profiling FBW7 and MCL1 protein levels in tumors could help predict a patient's response to antitubulin chemotherapeutics, Wertz said.

Her team plans to study the connection between FBW7 deficiency, increased MCL1 levels and cell survival in cancer cell lines and tumor models "to see how broad ranging the implications of our current study are."

Genentech declined to disclose the IP status of the team's findings, and Wertz said it is too early to say whether the results might lead Genentech to develop MCL1 inhibitors.

Abbott and Genentech are co-developing Navitoclax (ABT-263; RG7433) for solid tumors and hematological malignancies. Navitoclax is an orally available analog of ABT-737 that inhibits BCL2, BCL- $\mathrm{X}_{\mathrm{L}}$ and BCL2-like 2 (BCL2L2; BCLW).

\section{Zeroing in on MCL1}

Eutropics is developing undisclosed inhibitors of BCL2 family proteins, including MCL1, to treat myeloma, leukemia and other cancers. The company has exclusive rights to profiling technology from DanaFarber in order to identify MCL1-dependent cancers and "answer the question of what cancers are the best targets for MCL1 inhibition," said Letai, who invented the technology.

Letai is an assistant professor of medicine at Harvard Medical School and Dana-Farber but was not involved in Wei's paper. He told SciBX that resistance to BCL2-inhibiting compounds such as ABT-737 has provided an impetus for finding an effective inhibitor of MCL1.

Lance Leopold, CMO and VP of clinical development at Ascenta Therapeutics Inc., concurred with Letai. "Our published studies show that AT-101 is synergistic with ABT-737 in cell lines expressing MCL1," he said. Ascenta's AT-101, a small molecule pan-inhibitor of BCL2 family proteins, including MCL1, is in Phase II testing to treat multiple cancers. It is partnered with Ascentage Pharma Group Corp.

Moreover, Leopold said that published studies by the company's collaborators at the University of Pennsylvania have demonstrated that MCL1 expression is a predictor of AT-101 activity in neuroblastoma. ${ }^{8,9}$

Nevertheless, Leopold cautioned that "MCL1 protein expression alone is not the sole determinant of response to" BCL2 inhibitors such as ABT737. He said this is because cancer cells can express both proapoptotic and/or antiapoptotic proteins of the BCL2 family. Consequently, he said, inhibiting a single antiapoptotic protein such as MCL1 or BCL2 might not induce apoptosis unless the cancer also expresses a proapoptotic protein member of the BCL2 family such as BCL2-associated X protein (BAX) or BCL2-antagonist/killer 1 (BAK1).

Thus, Leopold and Letai agreed that further studies need to profile the expression of FBW7, MCL1 and other BCL2 family proteins in a broad range of cancer types to assess the therapeutic potential of both teams' findings.

Haas, M.J. SciBX 4(11); doi:10.1038/scibx.2011.300

Published online March 17, 2011

\section{REFERENCES}

1. Inuzuka, H. et al. Nature; published online March 2, 2011; doi:10.1038/nature09732

Contact: Wenyi Wei, Beth Israel Deaconess Medical Center, Boston, Mass. e-mail: wwei2@bidmc.harvard.edu

2. Wertz, I.E. et al. Nature; published online March 2, 2011; doi:10.1038/nature09779

Contact: Ingrid E. Wertz, Genentech Inc., South San Francisco, Calif. e-mail: ingrid@gene.com

3. Maser, R.S. et al. Nature 447, 996-971 (2007)

4. Matsuoka, S. et al. Genes Dev. 22, 986-991 (2008)

5. Wood, L.D. et al. Science 318, 1108-1113 (2007)

6. Tse, C. et al. Cancer Res. 68, 3421-3428 (2008)

7. Youle, R.J. \& Strasser, A. Nat. Rev. Mol. Cell Biol. 9, 47-59 (2008)

8. Lestini, B.J. et al. Cancer Biol. Ther. 8, 1587-1595 (2009)

9. Goldsmith, K.C. et al. Cell Death Differ. 17, 872-882 (2010)

COMPANIES AND INSTITUTIONS MENTIONED

Abbott Laboratories (NYSE:ABT), Abbott Park, III.

Ascenta Therapeutics Inc., Malvern, Pa.

Ascentage Pharma Group Corp., Hong Kong, China

Bayer AG (Xetra:BAY), Leverkusen, Germany

Beth Israel Deaconess Medical Center, Boston, Mass.

Brigham and Women's Hospital, Boston, Mass.

Dana-Farber Cancer Institute, Boston, Mass.

Eutropics Pharmaceuticals Inc., Dorchester, Mass.

Genentech Inc., South San Francisco, Calif.

Harvard Medical School, Boston, Mass.

The Jackson Laboratory, Bar Harbor, Me.

Kyushu University, Fukuoka, Japan

Massachusetts General Hospital Cancer Center,

Charlestown, Mass.

Onyx Pharmaceuticals Inc. (NASDAQ:ONXX), Emeryville, Calif.

Roche (SIX:ROG; OTCQX:RHHBY), Basel, Switzerland

The University of Melbourne, Parkville, Victoria, Australia

University of Pennsylvania, Philadelphia, Pa.

The Walter and Eliza Hall Institute of Medical Research,

Parkville, Victoria, Australia 